\title{
Heat flux distribution beneath evaporating hydrophilic and superhydrophobic droplets
}

\author{
M.J. Gibbons ${ }^{\mathrm{a}, *}$, P. Di Marco ${ }^{\mathrm{b}}$, A.J. Robinson ${ }^{\mathrm{a}}$ \\ ${ }^{a}$ Department of Mechanical, Manufacturing and Biomedical Engineering, Trinity College Dublin, the University of Dublin, Dublin 2, \\ Ireland. \\ ${ }^{b}$ DESTEC, University of Pisa, Italy.
}

\begin{abstract}
An experimental investigation of the heat and mass transfer to an evaporating hydrophilic water droplet using thin-foil thermography and droplet shape analysis is reported. These results have been compared with that of a superhydrophobic evaporating droplet. The hydrophilic droplet initially evaporated with a pinned contact line before unpinning and evaporating with a receding contact line. The largest heat flux is observed at the contact line region for both droplets. The hydrophilic droplet evaporated $34 \%$ faster than its superhydrophobic counterpart due to its greater contact line length, liquid-gas interface temperature and solid-liquid surface area for the majority of its evaporation. In general, the hydrophilic droplet dissipated a greater total power due to its larger contact line length and solid-liquid surface area, while the superhydrophobic droplet had a greater average heat flux due to its larger contact line length density for the majority of its evaporation time. The average heat flux to the evaporating droplets was demonstrated to vary as a linear function of the contact line length density.
\end{abstract}

Keywords: Heat transfer, Contact line, Droplet evaporation, Hydrophilic, Superhydrophobic

\section{Introduction}

Droplet evaporation represents a common natural phenomenon that has applications in numerous industrial fields: DNA mapping, ink-jet printing, surface patterning and evaporative spray cooling among others [2-14]. Heat and mass transfer to the contact line region of an evaporating droplet is a classical heat and mass transfer problem that is not yet fully solved. The thermal-physics of the threephase contact line is one area that still needs significant research in order to fully understand droplet evaporation. Albeit a proportionately small region compared with the overall droplet size, the wetting physics at the contact line are crucial in defining the static and dynamic mechanics and related heat and mass transfer of evaporating droplets. For example, depending on the wetting properties of the fluid and solid, it is possible to categorize four regimes of droplet evaporation: constant contact angle (CCA), constant contact radius (CCR), mixed, and stick-slip [15].

The characteristic difference between a hydrophilic and hydrophobic droplet is in the formation of their respective contact lines. For a hydrophilic droplet, the adsorbed film region forms due to the strong long-range intermolecular forces between the solid and liquid phases. These forces result in a contact line region incorporating several length scales (Fig. 1a) varying from nanometer in the adsorbed

\footnotetext{
* Corresponding author

Email address: Michael.Gibbons@tcd.ie (M.J. Gibbons)
}

film (10 - $20 \mathrm{~nm}[16])$ to the millimetre in the bulk droplet. The disjoining pressure in the adsorbed film prevents evaporation in this region [17]. As the film grows into the transition region, a reduction in the adhesion forces enables the highest local heat fluxes $[16,18]$ due to the low thermal resistance from the small film thickness $(1-3 \mu \mathrm{m})$ [16]. Moving radially inward from the transition to the microconvection region, the droplet film thickness grows, the local thermal resistance increases and the heat flux density decreases. Heat transfer within the central bulk of the droplet can be dominated by convection or conduction depending on the Péclet number. This contact line structure is distinctly different compared to a non-wetting droplet which experiences weak adhesion forces between the liquid and solid phases. This results in a considerably smaller adsorbed film region [13] which prevents the creation of a transition region, as illustrated in Fig. 1b. As a consequence, the contact line heat transfer mechanisms for a non-wetting droplet are substantially different to that of a wetting droplet. For an evaporating superhydrophobic droplet, the peak local heat flux has recently been observed at the contact line [15]. This is due to the highest liquidgas surface temperature occurring at the droplet base at the contact line. The heated surface establishes a thermal boundary layer in the adjacent liquid and the thermal resistance and heat flux density from the substrate is strongly influenced by this boundary layer. At the contact line, the boundary layer intersects the liquid-gas interface creating a region below which the liquid-gas surface tem- 


\section{Nomenclature}

\begin{tabular}{|c|c|c|c|}
\hline$A$ & Area $\left[\mathrm{mm}^{2}\right]$ & $b$ & base \\
\hline$C$ & Curvature $\left[\mathrm{mm}^{-1}\right]$ & $c$ & hydrophobically coated substrate \\
\hline$C_{p}$ & Specific heat capacity $[\mathrm{J}]$ & cap & Capacitive or storage \\
\hline$E$ & Energy $\left[\mathrm{W} \mathrm{m}^{-1} \mathrm{~K}^{-1}\right]$ & con & Heat transfer from substrate \\
\hline$H$ & height $[\mathrm{mm}]$ & cond & Conduction \\
\hline$k$ & Thermal conductivity $\left.\left[\mathrm{W} \mathrm{m}^{-1} \mathrm{~K}^{-1}\right]\right]$ & $d$ & Droplet \\
\hline$q^{\prime \prime}$ & Heat flux $\left[\mathrm{W} \mathrm{m}^{-2}\right]$ & $f$ & Foil \\
\hline$R$ & Radius $[\mathrm{mm}]$ & gen & Generated \\
\hline$S$ & Coordinate $[\mathrm{mm}]$ & $l c$ & Lateral conduction \\
\hline$T$ & Temperature $[\mathrm{K}]$ & $\lg$ & liquid-gas \\
\hline$t$ & Time $[\mathrm{min}]$ & $p$ & Paint \\
\hline$V$ & Volume $[\mu \mathrm{L}]$ & $r$ & Radial coordinate direction \\
\hline & & $s$ & Heated substrate \\
\hline \multicolumn{2}{|c|}{ Dimensionless Numbers } & $s l$ & solid-liquid \\
\hline$B i$ & $B i=h L / k[-]$ & $T$ & apex \\
\hline \multirow{2}{*}{\multicolumn{2}{|c|}{ Greek Symbols }} & $u$ & uncoated substrate \\
\hline & & $w f$ & Working fluid \\
\hline$\delta$ & Thickness $[\mu \mathrm{m}]$ & $x, y, z$ & Coordinate direction \\
\hline$\gamma$ & Surface tension $\left.\left[\mathrm{N} \mathrm{m}^{-1}\right]\right]$ & & \\
\hline$\rho$ & Density $\left[\mathrm{kg} \mathrm{m}^{-3}\right]$ & \multicolumn{2}{|c|}{ Acronyms } \\
\hline \multirow[t]{2}{*}{$\theta$} & Contact angle $\left[^{\circ}\right]$ & $\mathrm{CCA}$ & Constant contact angle \\
\hline & & CCR & Constant contact radius \\
\hline \multicolumn{2}{|c|}{ Subscripts } & CLD & Contact line length density \\
\hline$\infty$ & Bulk or infinity & IR & Infrared \\
\hline$a g$ & Air gap & $\mathrm{PU}$ & Percentage uncertainty \\
\hline
\end{tabular}

(a)

(b)
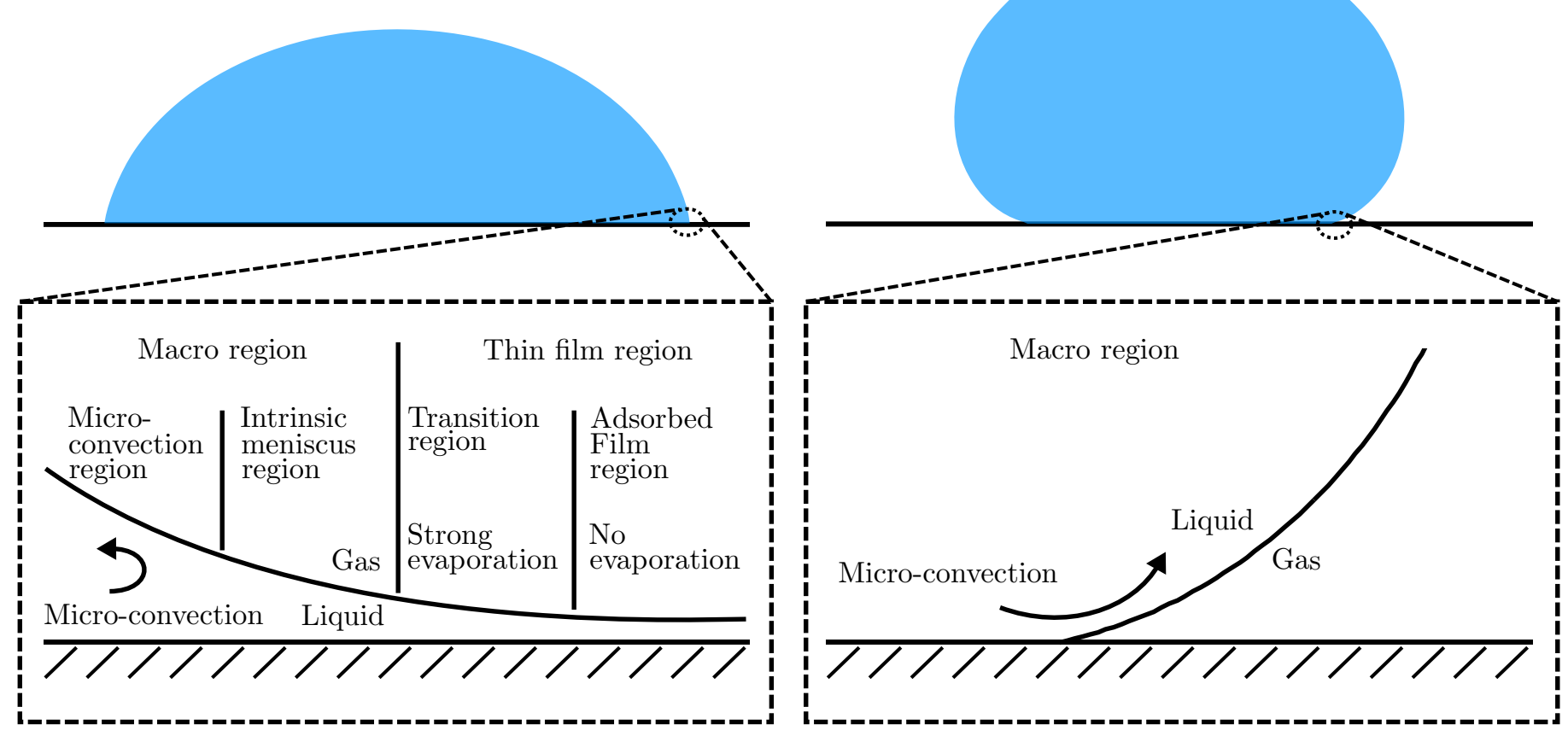

Figure 1: Evaporation at the contact line. (a) partially wetting droplet [1] and (b) partially non-wetting droplet. 


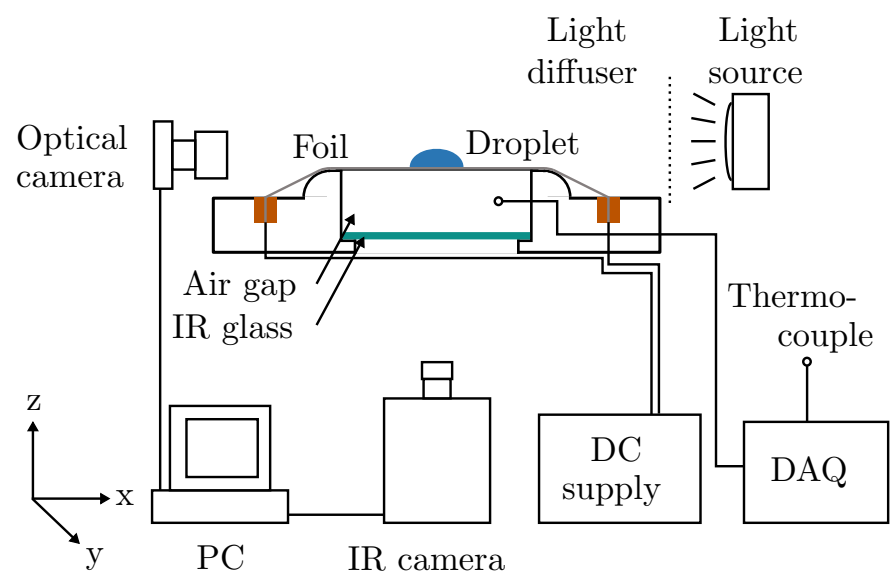

Figure 2: Schematic of the experimental apparatus.

perature is high. At this location, diffusion and possibly advection of heat to the droplet interface is released effectively, due to the proportionately high saturation pressure. Therefore, the close proximity of the heated surface and the liquid-gas interface at the contact line region combined with the high vapour diffusion to the ambient creates an overall lower thermal resistance pathway between the heat source (heated substrate) and the heat sink (the ambient air). Thus, contact line evaporation for a partially nonwetting droplet is also greatest at the contact line though due to quite different mechanisms compared with wetting droplets. Therefore, it can be said that the underlying physical mechanics of contact line evaporation for partial wetting and partial non-wetting droplets are now known, but there is substantial research required to fully understand their nature.

Previous work by the present authors [15] detailed the local heat flux distribution beneath an evaporating superhydrophobic water droplet using thermal imaging and geometric analysis of the evaporating droplet. The current investigation extends upon this earlier work by exploring the spatial distribution of the surface heat flux beneath an evaporating hydrophilic droplet over its complete life-cycle and comparing it to that of the previously characterized superhydrophobic droplet. To the best of knowledge, this study is the first to compares the local heat transfer beneath both a partially non-wetting and partially wetting evaporating droplets for a full evaporation event. Simultaneously, the droplet interfaces are analyzed and contrasted to investigate the impact of geometric properties on heat transfer and droplet mechanics.

\section{Experiment and Analysis}

\subsection{Experimental apparatus}

The experimental facility, data reduction and analysis have been described in detail in previous work published by the present authors [15]. Therefore only a brief description will be given here. The apparatus is outlined in
Table 1: Foil and paint properties [15, 19].

\begin{tabular}{lll}
\hline & Foil & Paint \\
\hline Thickness, $\delta[\mu \mathrm{m}]$ & 25 & 10.52 \\
Density, $\rho\left[\mathrm{kg} \mathrm{m}^{-3}\right]$ & 7960 & 1261 \\
Thermal conductivity, $k$ & 16.3 & 0.095 \\
{$\left[\mathrm{~W} \mathrm{~m}^{-1} \mathrm{~K}^{-1}\right]$} & 502 & 2835 \\
Specific heat, $\mathrm{C}_{p}\left[\mathrm{~J} \mathrm{~kg}^{-1} \mathrm{~K}^{-1}\right]$ & 60 & - \\
Roughness uncoated, $R a_{u}[\mathrm{~nm}]$ & 176 & - \\
Roughness coated, $R a_{c}[\mathrm{~nm}]$ & - & 0.95 \\
Paint emissivity, $\varepsilon_{p}$ & & \\
\hline
\end{tabular}

Fig. 2 and can be divided into two key parts; the heat transfer section and the imaging system. The resistively heated substrate (Fig. 2) is a $25 \mu \mathrm{m}$ thick 316 stainless steel foil (Goodfellow, $140 \times 80 \times 0.025 \mathrm{~mm}^{3}, \mathrm{P} / \mathrm{N}: 505-$ 400-04). The underside of the foil is coated with a $10.5 \mu \mathrm{m}$ thick layer of matte black paint to provide a known, high emissivity for the infrared thermography. The substrate properties are outlined in Table $1[15,19]$. Two top surface substrate conditions are implemented; hydrophilic and superhydrophobic. The hydrophilic condition corresponds to the raw uncoated steel foil, while the superhydrophobic case is achieved using Glaco Mirror Coat Zero [15]. The thickness and thermal resistance of the coating are assumed sufficiently thin and negligible respectively during the analysis of the experimental data. The wetting properties of the hydrophilic and superhydrophobic droplets are listed in Table 2.

The imaging system is composed of an optical camera (Point Grey, P/N: CMLN-1352M) and a high speed, highresolution thermal imaging camera (FLIR P/N: SC6000). Data acquisition for both cameras is controlled by a purposebuilt LabVIEW program. The optical camera is mounted parallel to the steel foil and is focused on the side profile of the evaporating droplet. It has a resolution of $1280 \times 960$ pixels with a pixel size of $10.5 \mu \mathrm{m}$. The thermal imaging camera is focused on the underside of the steel foil and captures the temperature distribution beneath the evaporating droplet. During testing, the thermal imaging camera records at a resolution of $400 \times 400$ pixels, with each pixel having a spatial resolution of $160 \mu \mathrm{m}$ and a noise equivalent differential temperature of $<20 \mathrm{mK}$.

Experiments are conducted under ambient conditions. An $80 \mu \mathrm{L}$ water droplet is deposited on the substrate at the start of testing. The foil is heated by Joule effect producing by a uniform volumetric heat generation and a subsequent average surface heat flux of $912 \mathrm{~W} \mathrm{~m}^{-2}$, this results in a substrate temperature of $\sim 70^{\circ} \mathrm{C}$ at steady state when no droplet is present. Due to the low Biot numbers, the temperature through the thickness of the foil and paint layers is assumed constant. Thermal data is acquired for 1 second every 60 seconds, the Point Grey optical camera is set to continuously record at $2 \mathrm{~Hz}$ throughout the evaporation period. 


\begin{tabular}{lccc}
\hline & Hydrophilic & Superhydrophobic & \\
\hline Advancing contact angle, $\theta_{A}$ & 85 & 160 & {$\left[^{\circ}\right]$} \\
Receding contact angle, $\theta_{R}$ & 50 & 159 & {$\left[^{\circ}\right]$} \\
\hline
\end{tabular}

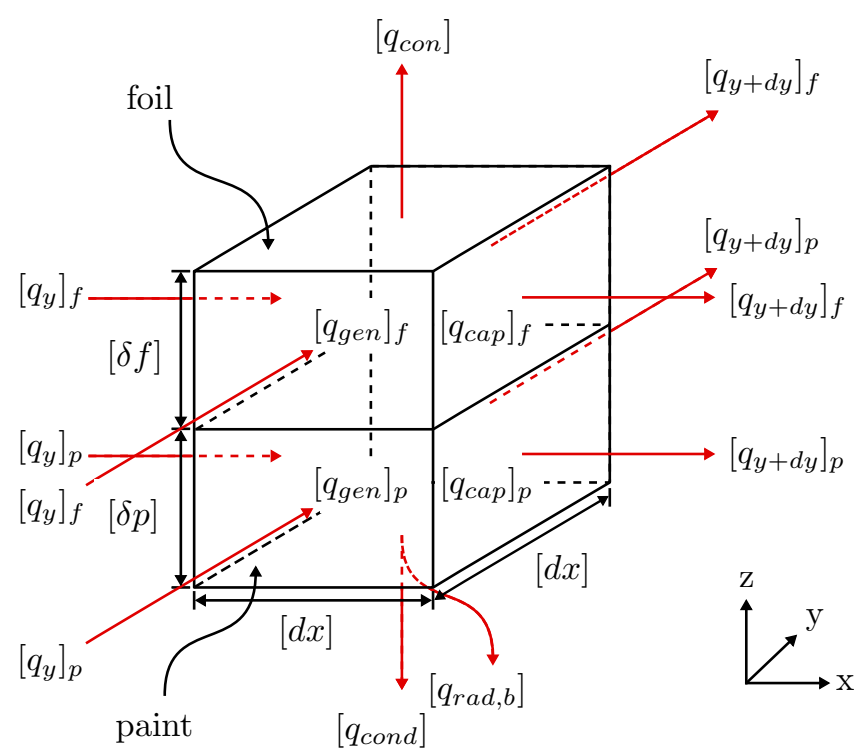

Figure 3: Heated substrate energy flow balance within a single dx $\mathrm{x}$ $\mathrm{dx}$ element of thickness $\delta_{f}$ and $\delta_{p}$.

\subsection{Analysis}

To determine the heat transfer and interfacial dynamics of the droplet both the spatial temperature distribution and the captured optical image of the evaporating droplet are analysed. All experimental data is processed in MATLAB. To calculate the thermal energy convected to the droplet an element-wise energy balance is preformed on the substrate, with each element consisting of a volume of $d x \times d x \times \delta$, where $d x$ is the pixel width of the IR camera and $\delta$ is the thickness of the substrate. Fig 3 illustrates the heat transfer energy flow within a singly element of the heated substrate. Uniform heat generation across the foil and paint layers is assumed. A lumped capacitance analysis is preformed as the Biot number is $\ll 1$ for both the foil and paint layers. Accounting for system losses, conjugate heat transfer and energy storage within the substrate yields:

$$
\begin{aligned}
q_{d}^{\prime \prime}=q_{g e n}^{\prime \prime}- & q_{\text {cond }}^{\prime \prime}-q_{\text {rad }, b}^{\prime \prime} \\
+\left(k_{f} \delta_{f}+\right. & \left.k_{p} \delta_{p}\right)\left(\frac{\partial^{2} T_{s}}{\partial x^{2}}+\frac{\partial^{2} T_{s}}{\partial y^{2}}\right) \\
& \quad-\left(\rho_{f} C_{p, f} \delta_{f}+\rho_{p} C_{p, p} \delta_{p}\right) \frac{\partial T_{s}}{\partial t}
\end{aligned}
$$

where $k_{f}, k_{p}, \delta_{f}, \delta_{p}, \mathrm{C}_{p, f}, \mathrm{C}_{p, p}$ are the foil and paint thermal conductivity, thickness and specific heat capacity respectively. Values for these parameters are shown in Table 1. Eq. 1 accounts for the generated flux within the

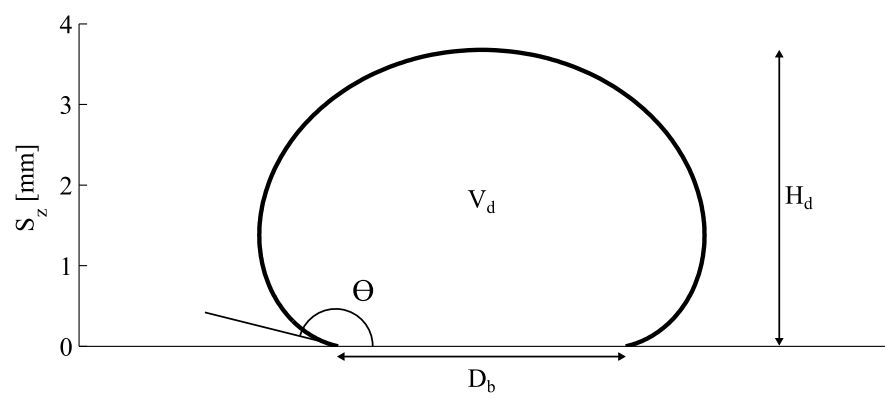

Figure 4: Schematic of droplet geometric parameters.

substrate $\left(q_{\text {gen }}^{\prime \prime}\right)$, the one-dimensional conduction $\left(q_{\text {cond }}^{\prime \prime}\right)$ and the radiation $\left(q_{\text {rad,b }}^{\prime \prime}\right)$ through the $7 \mathrm{~mm}$ air gap from the underside of the substrate. The final two terms in Eq. 1 are the heat transfer due to lateral conduction $\left(q_{l c}^{\prime \prime}\right)$ and heat storage $\left(q_{\text {cap }}^{\prime \prime}\right)$ within the substrate respectively. $q_{\text {con }}^{\prime \prime}$ is the heat flux transferred from the heated substrate into the evaporating droplet. $q_{c o n}^{\prime \prime}$ also encompasses the heat flux into the surrounding air in the far-field $\left(S_{r}>R_{b}\right)$. The droplet evaporation is assumed quasi-steady during the 1 second capture period. This approximation is correct due to the proportionately small calculated energy storage term for all test points. The radial profile of the heat flux is determined by averaging lines taken radially from the centre of the droplet at $0.5^{\circ}$ increments.

The interface profile of the evaporating droplet is determined from the captured optical images. From the droplet interface a number of key geometric parameters can be calculated, these are illustrated in Fig. 4. During evaporation, the droplet boundary at the contact line can become obscured due to thermal refraction, shadowing and reflections. Using the droplet height, approximate droplet volume and apex curvature it is possible to calculate the evaporating droplet interface and thus contact angle using the Young-Laplace equation for the present hydrostatic case [20], this is given by:

$$
\gamma\left(\frac{1}{R_{1}}+\frac{1}{R_{2}}\right)=\frac{2 \gamma}{R_{T}}-g\left(\rho_{l}-\rho_{g}\right)\left(z-H_{d}\right)
$$

where $R_{1}$ and $R_{2}$ are the principal radii of curvature, $R_{T}$ is the radius of the droplet apex, $\gamma$ is the surface tension, $\rho_{l}$ and $\rho_{g}$ are the density of the liquid and surrounding gas respectively, $H_{d}$ and $z$ are the droplet height and the height of the point on the interface being evaluated.

\subsection{Uncertainty}

The propagation of errors in the calculated thermal and geometric values are determined using the Taylor se- 

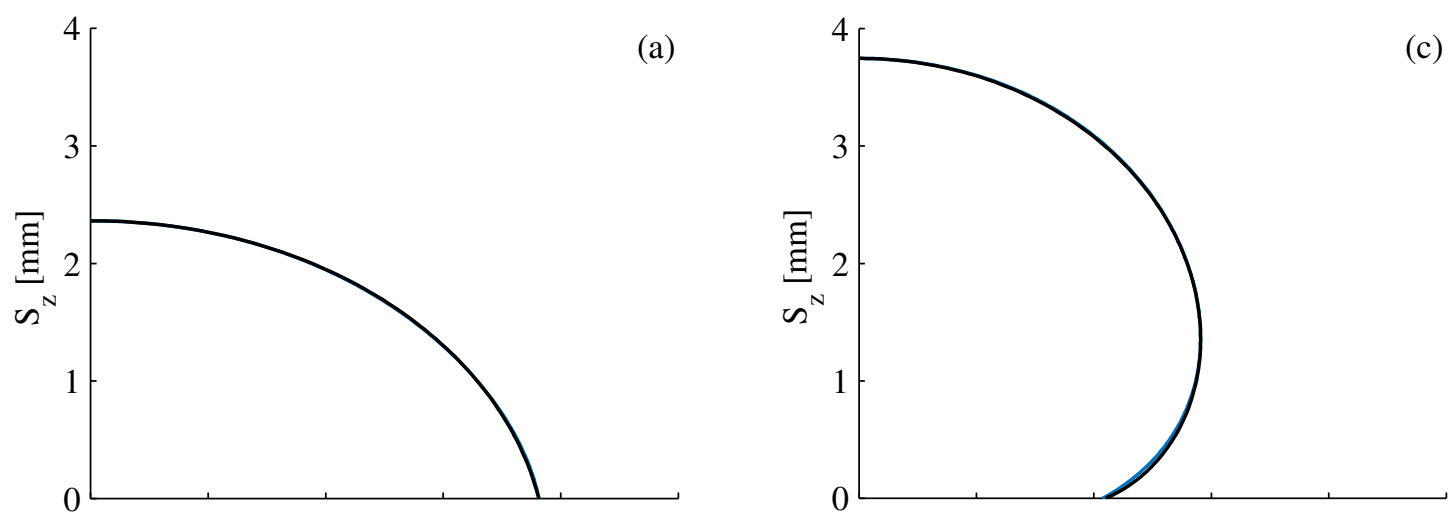

(c)
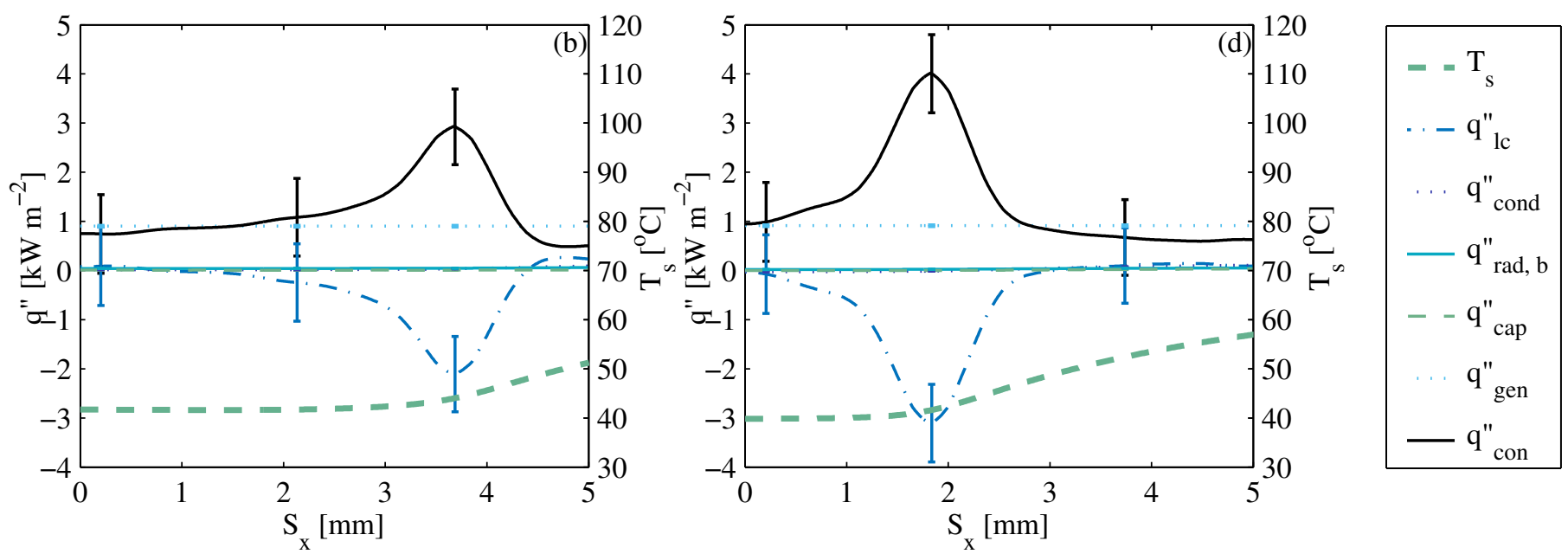

Figure 5: Hydrophilic ( $\mathrm{a}-\mathrm{b})$ and superhydrophobic $(\mathrm{c}-\mathrm{d})$ droplet profile and energy balance at $\mathrm{t}=2 \mathrm{~min}$.

Table 3: Experimental uncertainty.

\begin{tabular}{lcr}
\hline Parameter & & $\mathrm{PU}[\%]$ \\
\hline Substrate temperature, $T_{s}$ & \pm & 0.26 \\
Ambient temperature, $T_{\infty}$ & \pm & 0.05 \\
Air gap temperature, $T_{a g}$ & \pm & 0.04 \\
Generated flux, $q_{g e n}^{\prime \prime}$ & \pm & 1.95 \\
Droplet volume, $V_{d}$ & \pm & 5.21 \\
Contact angle, $\theta$ & \pm & 35.19 \\
Base radius, $R_{b}$ & \pm & 2.66 \\
Droplet height, $H_{d}$ & \pm & 1.51 \\
Apex radius, $R_{T}$ & \pm & 2.68 \\
\hline
\end{tabular}

ries method [21] and their values are provided in Table 3. The applied method takes into consideration the uncertainties of the calibration curves fitted to thermal data, the precision and accuracy of the voltage, current and optical measurements. Correlation and covariance of the thermal data are accounted for through crosscorrelation and autocorrelation for the lateral conduction and energy storage terms respectively. Fig. 5b and d present the individual contributions of all the energy balance terms for both the partial non-wetting and partial wetting droplets. The associated uncertainty of these terms is shown at three locations on the radial profile. These values are predominately a function of the noise of the IR camera InSb sensor. Each droplet case was tested three times; a good correlation of experimental data was noted for all cases.

\section{Results and Discussion}

Fig. 5 compares the liquid-gas interface (Fig. 5a and c) with the radial heat flux energy balance at $t=2 \mathrm{~min}$ (Fig. 5b and d) for both the hydrophilic and superhydrophobic droplets respectively. The peak heat transfer for both cases is positioned at the contact line, with the lateral conduction of heat having the greatest contribution to the energy balance. This is consistent with previously published droplet evaporation and spray cooling studies [1416, 18]. Heat transfer away from the contact line in the central bulk of the droplet can be through convection or conduction depending on the Péclet Number [22, 23]. The Péclet Number changes over the course of the evaporation of a droplet due to the changing size and temperature distribution within the droplet. As such, the flow field within 
Table 4: Droplet evaporation comparison at $t=2 \mathrm{~min}$.

\begin{tabular}{|c|c|c|c|}
\hline & Hydrophilic & Superhydrophobic & \\
\hline Volume, $V$ & 68.33 & 73.16 & $\mu \mathrm{L}$ \\
\hline Contact angle, $\theta$ & 74.2 & 156.2 & $\stackrel{0}{\circ}$ \\
\hline Base radius, $R_{b}$ & 3.85 & 2.09 & $\mathrm{~mm}^{2}$ \\
\hline Liquid-gas area, $A_{l g}$ & 67.4 & 74.3 & $\mathrm{~mm}^{2}$ \\
\hline Peak heat flux, $q_{\text {peak }}^{\prime \prime}$ & 2.87 & 4.00 & $\mathrm{~kW} \mathrm{~m}^{-2}$ \\
\hline Total droplet power dissipated, $\mathrm{q}_{\text {con }}$ & 80.43 & 42.02 & $\mathrm{~mW}$ \\
\hline
\end{tabular}

the central bulk of the droplet is not studied in this work, only the heat transfer from the substrate into the droplet is characterised. Table 4 compares key droplet properties of both cases at $t=2 \mathrm{~min}$. For similar droplet volumes and liquid-gas surface areas, the superhydrophobic droplet has a $39 \%$ larger peak heat flux in comparison to the hy-
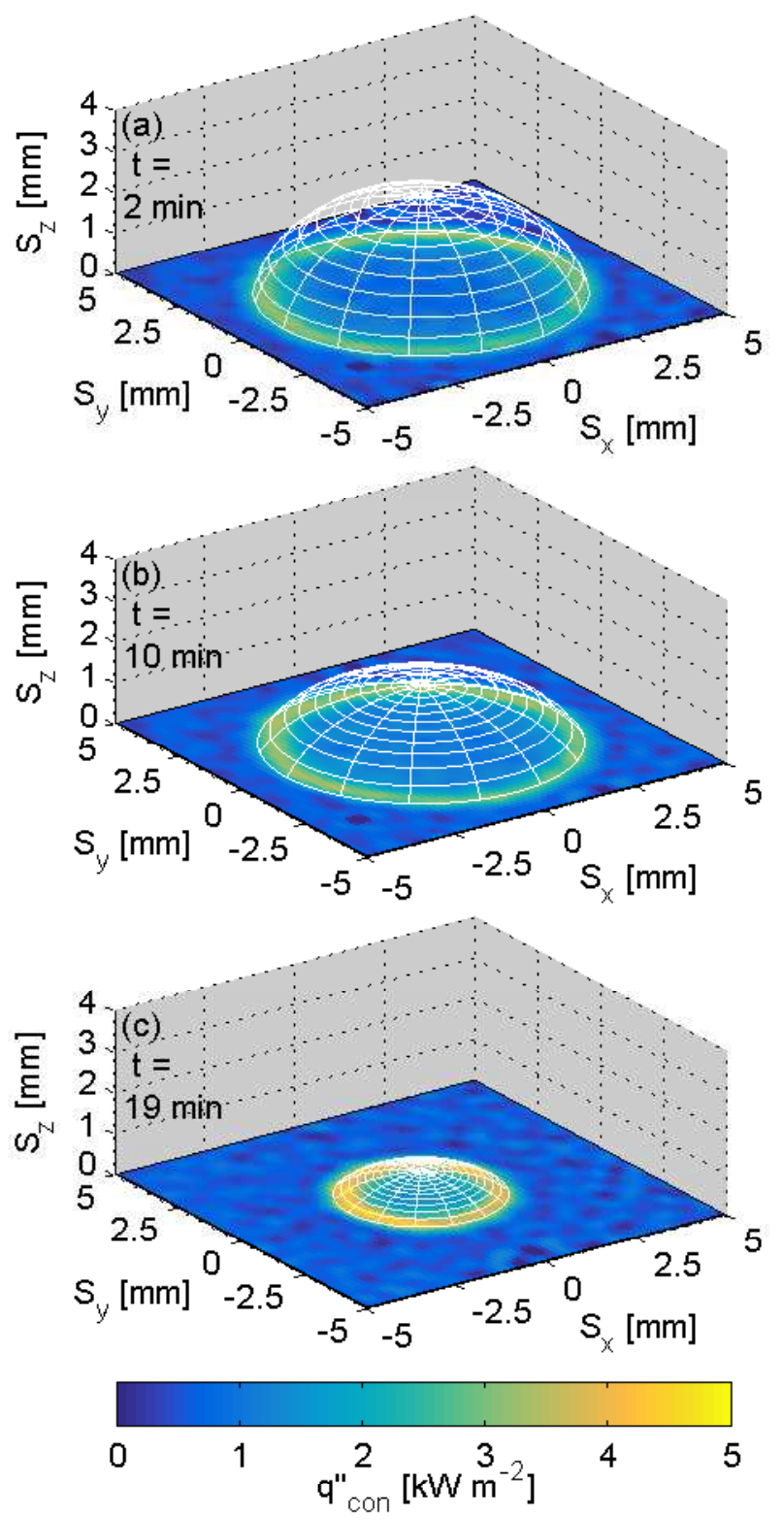

drophilic case. This is as a result of the larger contact line length density ${ }^{1}$ (CLD) [15] in the superhydrophobic case. In contrast, the hydrophilic droplet dissipates twice as much thermal power $\left(q_{c o n}\right)$ due to its larger solid-liquid

\footnotetext{
${ }^{1} \mathrm{CLD}=$ contact line length / wetted area
}
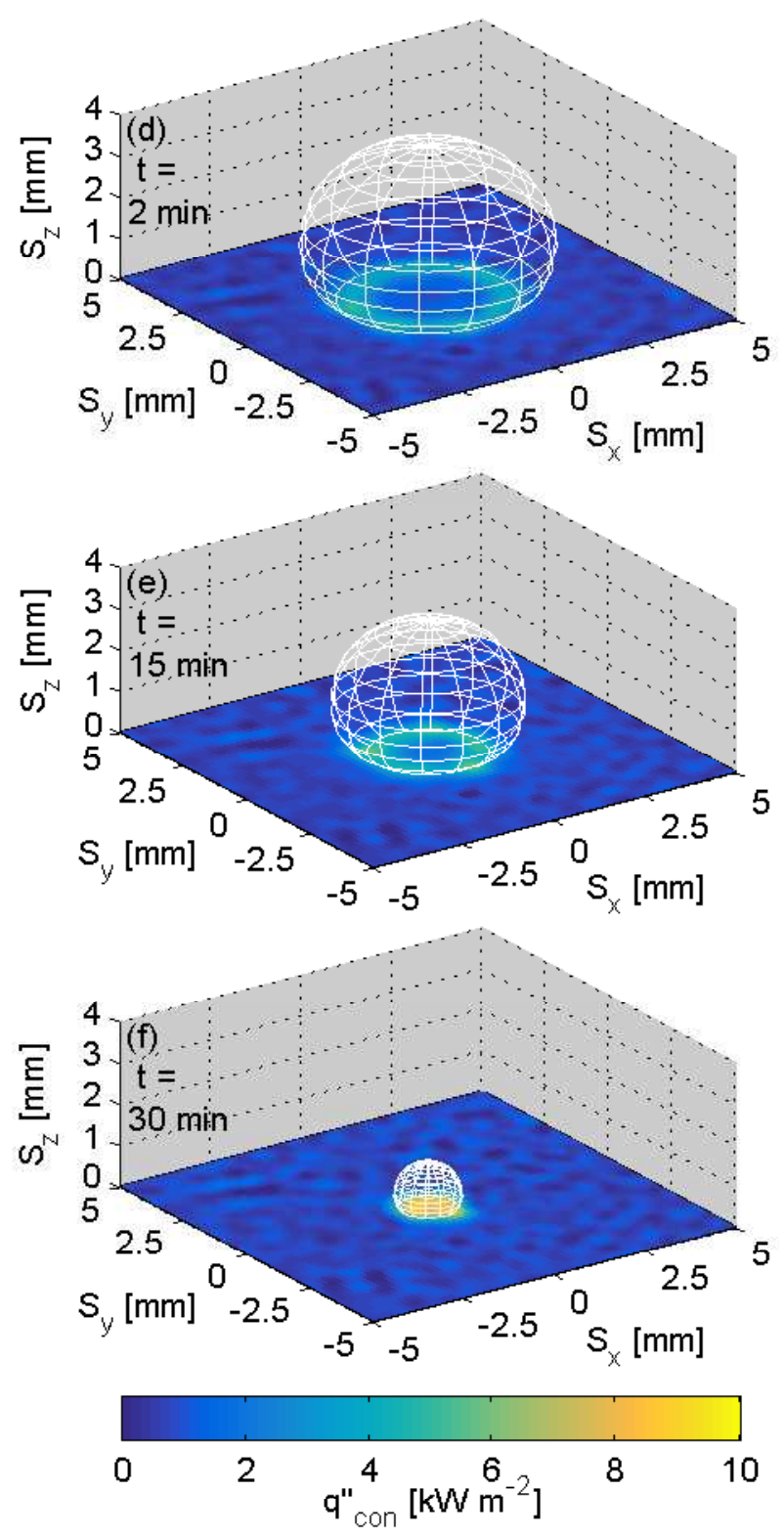

Figure 6: Hydrophilic (a - c) and superhydrophobic ( $\mathrm{d}-\mathrm{f})$ morphology and heat tansfer through time. 
surface area and total contact line length. This is clearer when comparing Fig. 6 a and d, which show the droplet geometries and heat flux footprints of the droplets corresponding to Fig. 5. It is clear that both the high heat flux contact line area and the lower heat flux central area are larger for the hydrophilic droplet owing to the higher surface energy which acts to spread the droplet to a larger base radius, resulting in a larger contact line circumference and base area. Thus, despite having similar volumes and liquid-gas surface areas the hydrophilic droplet dissipates a much larger thermal power.

Fig. 6 contrasts the evaporation modes, interface morphologies and heat flux distributions of the hydrophilic and superhydrophobic droplets at the beginning (Fig. 6a and d), middle (Fig. 6c and e) and end (Fig. 6c and f) of their evaporations. For convenience, these can be crossreferenced with the respective time-histories of their geometric properties, which are plotted in Fig. 7 for the
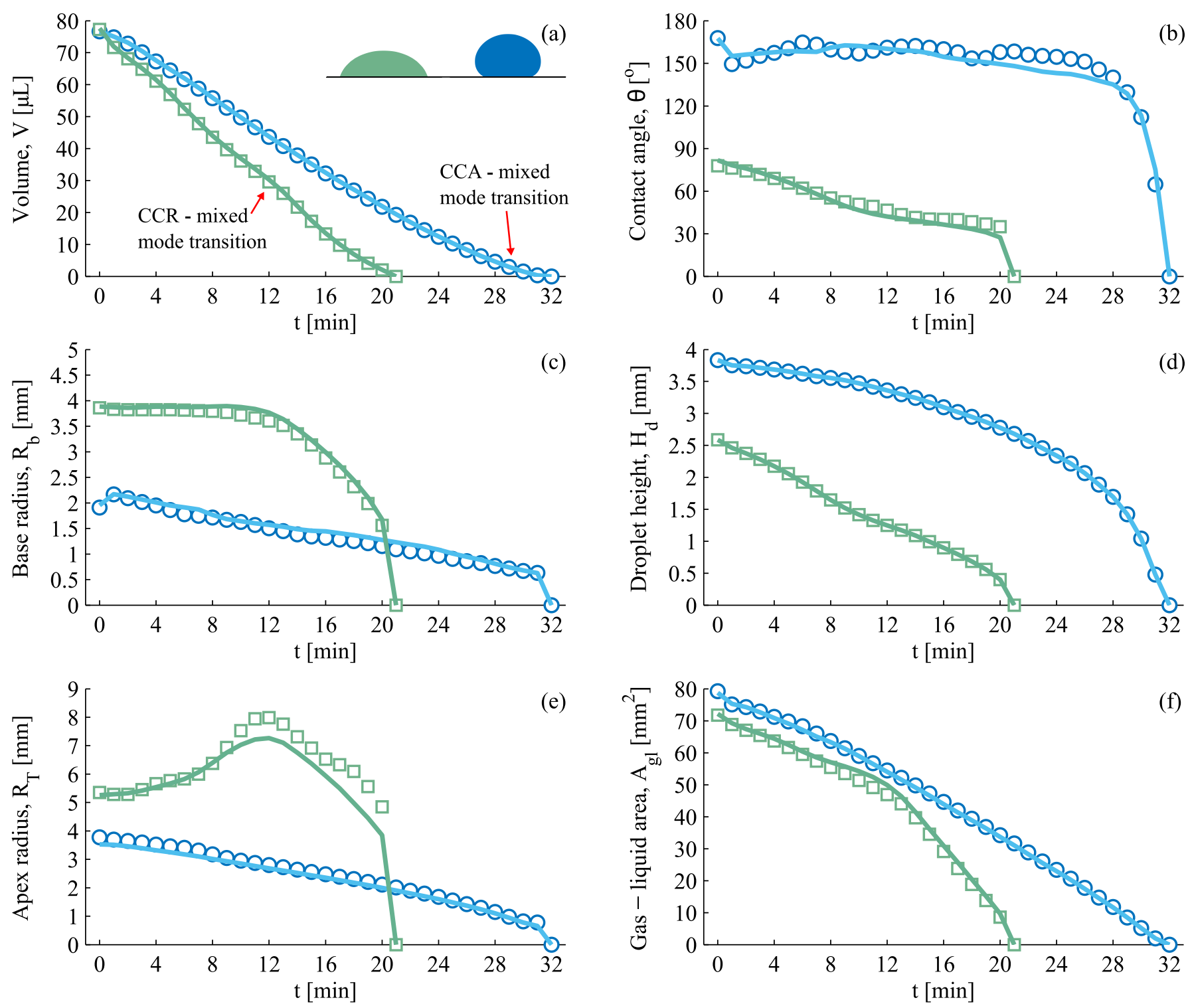

○ Superydrophobic measured

Superydrophobic theoretical
Hydrophilic measured

Hydrophilic theoretical

Figure 7: Hydrophilic and superhydrophobic droplet geometric properties through time. (a) volume, (b) contact angle, (c) base radius, (d) height, (e) apex principal radii, and (f) liquid-gas surface area. Empty markers = axisymmetric droplet, filled markers = non-axisymmetric droplet. 
entire evaporation periods. Measured and calculated values from the Young-Laplace solution are shown, with solid data points denoting when the axisymmetric assumption is not valid.

Referring to Fig. 7, the superhydrophobic droplet evaporates primarily in the constant contact angle mode. From $t=1-28 \mathrm{~min}$ the droplet evaporated with a relatively constant contact angle of $\theta \approx 150^{\circ}$, with a receding contact line and decreasing droplet height (Fig. $7 \mathrm{~d}-\mathrm{f})$. As the droplet approaches complete evaporation $(t=29-32$ min), it transitions into a mixed-mode regime. This mode makes up only $10 \%$ of the total evaporation time and is denoted by a decreasing contact angle and base radius. The evaporation path and evolution of geometric parameters are consistent with those previously reported by Dash and Garimella [24]. In contrast, the hydrophilic droplet evaporates initially in the CCR mode before transitioning into the mixed-mode regime. This is shown by the near-constant base radius, monotonically decreasing contact angle and droplet height from $t=0-11 \mathrm{~min}$. This is shown qualitatively in Fig. 7a and b. At $t=12$ min, the contact line becomes unpinned due to the contact angle decreasing to the receding contact angle and the droplet begins to evaporate in the mixed-mode regime (Fig. $7 \mathrm{~b}$ and c), with a decreasing base radius, contact angle and droplet height for $t=13-21 \mathrm{~min}$. These results agree with what has been previously observed in the literature [25-28] for pinned droplet evaporation on heated substrates.

Fig 8 a and $d$ shows the heat flux into both droplets over the course of their respective evaporation lifespans. As previously noted [15], the receding contact line of the superhydrophobic droplet results in an increased average and peak heat flux due to the escalating liquid-gas interface temperature and CLD. The receding nature of contact line also serves to mitigate the accumulation of vapour concentration adjacent to the contact line [29]. A decrease in the total thermal power dissipated is observed and this is due to the shrinking solid-liquid interface area and contact line length.

In terms of heat transfer, Figs. $8 \mathrm{a}$ and $\mathrm{b}$ illustrate some key differences between the superhydrophobic and hydrophilic droplets. In Fig. 8a it is clear that the peak heat flux at the contact line is notably lower for the hydrophilic case when evaporating in CCR mode $(t<11$ $\min )$. The fact that there is a difference is not surprising considering that, as discussed, the mechanisms of contact line heat transfer are quite different between superhydrophobic and hydrophilic droplets. However, there is
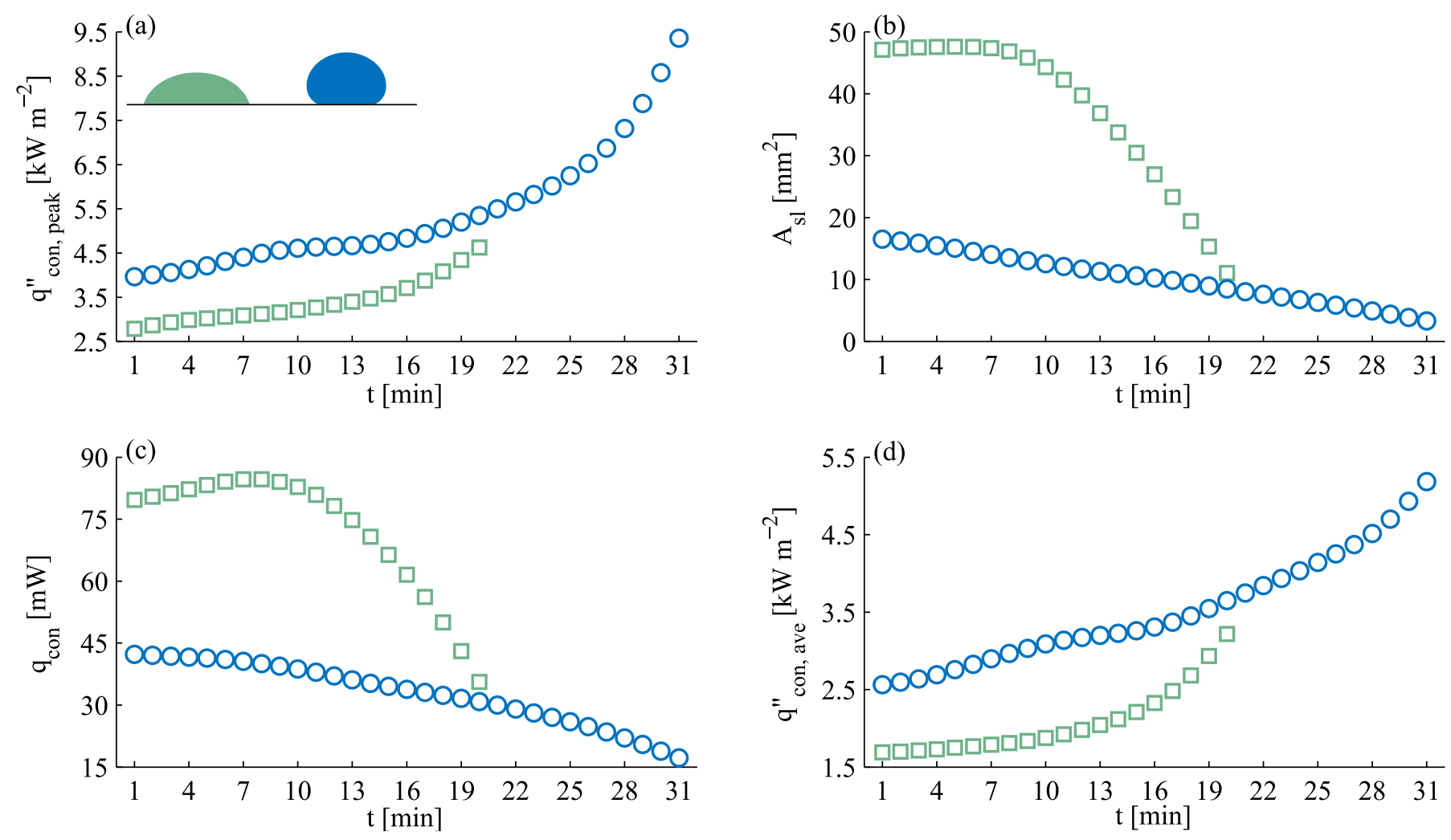

Superydrophobic measured

Hydrophilic measured

Figure 8: Hydrophilic and superhydrophobic droplet evaporation heat transfer. (a) peak heat flux, (b) solid-liquid surface area, (c) thermal power dissipated, and (d) average heat flux. 
insufficient detail here to explain exactly why the thin film micro-region of the partial-wetting droplet is less effective. It is noticed, however, that once the hydrophilic droplet de-pins at $t \sim 11 \mathrm{~min}$ and evaporates in a mixed, though predominantly CCA, mode (Fig. 7b), the contact line heat flux escalates. This is further evidence to support a general conclusion that CCR contact line evaporation is less intense than CCA evaporation, though the underlying reason will have to be studied further in the simulation environment.

Fig. 8b shows that during the CCR evaporation mode of the hydrophilic droplet $(t<11 \mathrm{~min})$, the solid-liquid contact area, that being the area through which thermal energy is transferred into the liquid from the heated base, is constant and significantly higher than that of the superhydrophobic droplet. This is important for the instantaneous effective thermal resistance of the droplet. The peak (Fig. 8a) and average (Fig. 8d) heat flux to the hydrophilic droplet are lower than that to the hydrophobic one. However, due to the pinning of the contact line, and subsequent much larger solid-liquid heat transfer surface area, the thermal resistance of the hydrophilic drop is considerably less resulting in about double the thermal power dissipation, as shown in Fig. 8c. Thus, the general conclusion is that for the same droplet volume, CCR mode evaporation creates the lower overall thermal resistance compared with CCA evaporation because of the higher liquid-solid surface area more than offsets the lower heat flux. This, combined with the larger contact line length, results in notably more thermal power being transferred to hydrophilic droplets compared with superhydrophobic ones of commensurate volume.

Once the hydrophilic droplet de-pins and it enters the mixed evaporation mode $(t=11-21 \mathrm{~min})$, the peak and average heat flux (Figs 8a and $\mathrm{d}$ ) escalate to the point that, at the end of its life, they are comparable with the levels observed for the hydrophobic droplet. Similarly, the thermal power decreases (Fig 8c) owing largely to the decrease in the wetted surface area (Fig 8b). This exposes another fundamental difference between the behaviour of the two droplet types, considering that the superhydrophobic droplet experiences an almost steady decrease in the wetted area and thermal power since its contact angle remains constant until the very last stage of its evaporation cycle (Fig. 7b).

The increase in the average heat flux was rationalized in Gibbons et al. [15] for the superhydrophobic droplet by considering the Contact Line Density (contact line length / wetted area). This value describes the relative magnitude of the contact line length, where high evaporative heat flux is observed, to the overall wetted heat transfer area. It was shown that the increasing CLD increases the average heat flux since the proportionately high contact line heat flux will be continually more influential over the respective contact area.

With respect to the hydrophilic versus superhydrophobic droplet comparison, the influence of the CLD is illus-

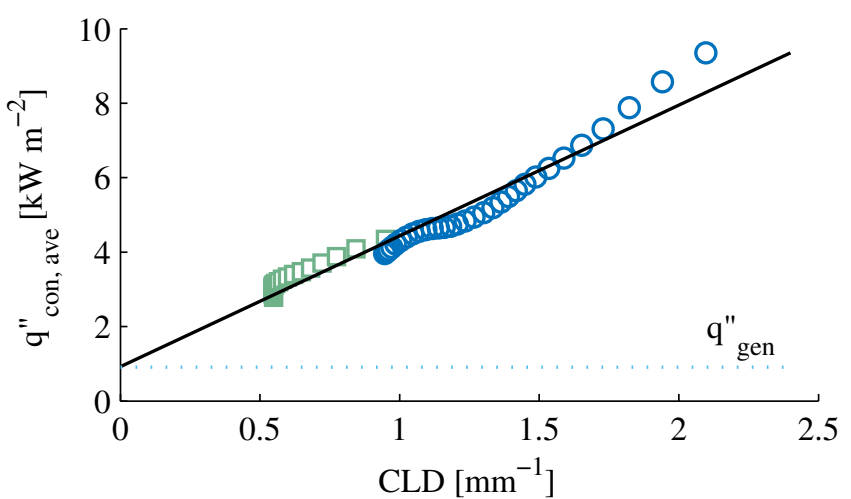

Figure 9: Average heat flux to the evaporating droplets compared with the contact line length density over the complete evaporation period.

trated in Fig. 5 for droplets of similar volume. For the pinned hydrophilic droplet the CLD $\sim 0.5 \mathrm{~mm}^{-1}$, and the average heat flux is $q_{\text {con, ave }}^{\prime \prime} \sim 1.7 \mathrm{~kW} \mathrm{~m}^{-2}$. This average heat flux is notably less than that of the superhydrophobic droplet $\left(q_{\text {con, ave }}^{\prime \prime} \sim 2.6 \mathrm{~kW} \mathrm{~m}^{-2}\right.$ ), because the larger base radius keeps the high heat flux contact line region far from the centre, resulting in a large interior region of 'low' heat flux i.e. comparable with the applied generated heat flux (Fig. 5b). Conversely, the superhydrophobic droplet has a significantly lower base radius resulting in a CLD $\sim 0.95$ $\mathrm{mm}^{-1}$ and it is clear from Fig. $5 \mathrm{~d}$ that the high contact line heat transfer, in this case, is influential over the entire heat transfer region of the droplet.

Fig. 9 plots the average heat flux to the droplets versus CLD for both the hydrophilic and superhydrophobic cases over their full evaporation periods. All of the data can be correlated by a single linear line that intersects the vertical axis at the applied heat flux. These illustrate some potentially key insights with regard to the CLD and the overall thermal resistance to heat transfer.

For an ideal symmetric droplet, CLD $=2 / R_{b}$. Thus, the vertical axis intercept is associated with the case where $\mathrm{R}$ is infinite. This is of course no longer a droplet, but that of evaporation across a liquid layer of constant thickness. Here there is no enhanced influence of the low thermal resistance contact line region, the heat flux is simply the applied heat flux, and the overall thermal resistance is a series sum of those associated with the liquid layer, the gasliquid interface and convection into the surrounding air. For a formed droplet, the heat transfer from the heated base to the ambient has two parallel pathways; one associated with the main dome (with mechanisms similar in nature to that just described above), and one via the contact line region. Decreasing $R_{b}$ progressively increases the CLD due to their inverse relationship. Physically, this progressively increases the proportion of the total heat transfer area that is influenced by the low thermal resistance contact line region, at the expense of that associated with the dome region. This is clear from the comparison of the heat flux profiles for the droplets in Fig. 5. In Fig. 6f, where 
Table 5: Droplet evaporation comparison for similar CLD.

\begin{tabular}{lccc} 
& Hydrophilic & Superhydrophobic & \\
\hline Contact line length density, CLD & 1.19 & 1.22 & $\mathrm{~mm}^{-1}$ \\
Volume, $V$ & 2.06 & 49.73 & $\mathrm{\mu L}$ \\
Contact angle, $\theta$ & 27.47 & 162.3 & $\mathrm{~mm}^{\circ}$ \\
Base radius, $R_{b}$ & 1.68 & 1.64 & 8.45 \\
Solid-liquid surface area, $A_{s l}$ & 8.87 & 58.96 & $\mathrm{~mm}^{2}$ \\
Liquid-gas surface area, $A_{l g}$ & 9.66 & 4.61 & $\mathrm{~mm}^{2}$ \\
Peak heat flux, $q_{\text {peak }}^{\prime \prime}$ & 4.62 & 3.09 & $\mathrm{~kW} \mathrm{~m}^{-2}$ \\
Average heat flux, $q_{\text {ave }}^{\prime \prime}$ & 3.22 & $\mathrm{~kW} \mathrm{~m}^{-2}$ \\
\hline
\end{tabular}
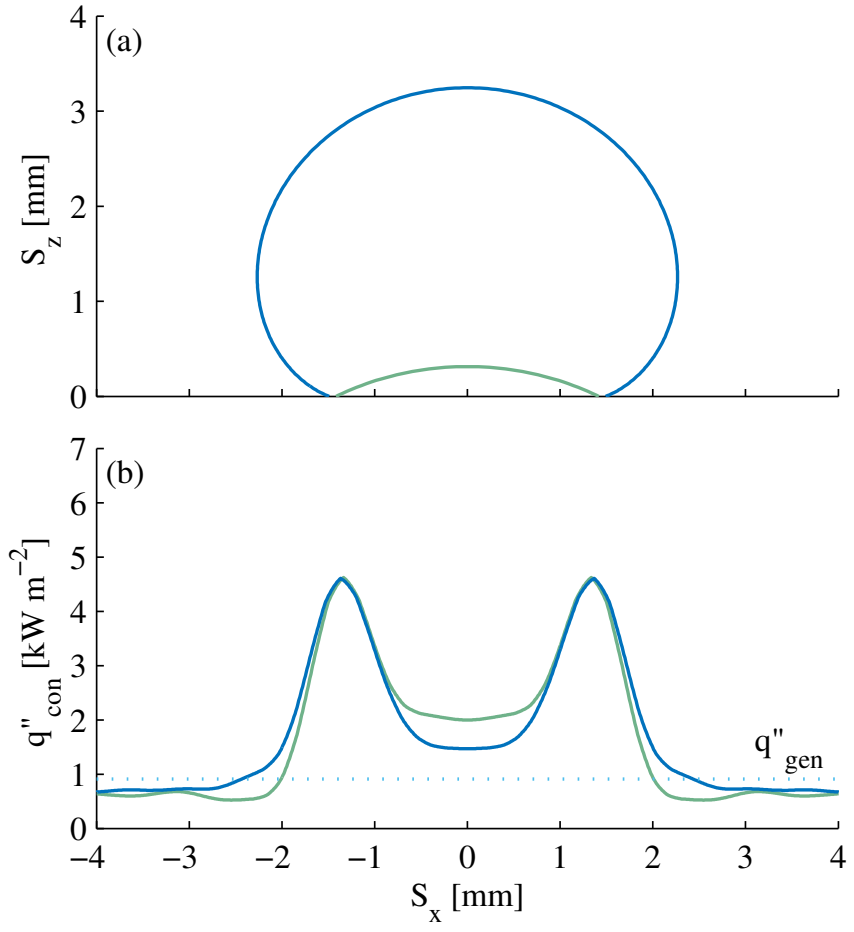

Figure 10: Hydrophilic and superhydrophobic droplet evaporation for a similar CLD. (a) liquid-gas interface and (b) heat flux profile.

$R_{b} \sim 0.67 \mathrm{~mm}, \mathrm{CLD} \sim 3 \mathrm{~mm}^{-1}$, the heat transfer is now in fact dominated by the contact line over the entire base area.

Simply put, as the CLD increases, the proportion of the overall thermal resistance associated with transporting energy to the ambient via the droplet dome decreases, whilst that associated with the contact line region increases. Importantly, this generalized effect should be independent of the thermal boundary condition beneath the droplet. Considering the two idealized extremes, a constant temperature base should experience an increased average heat flux with decreased CLD, whilst a constant heat flux base should experience a decrease in the average temperature.

Fig. 10 compares both evaporation cases for a constant CLD. These correspond to $t=10 \mathrm{~min}$ and $t=20 \mathrm{~min}$ for the superhydrophobic and hydrophilic droplets respectively and represents the region where the CLD of the two data sets overlap. Table 5 compares droplet properties of both cases. Despite the large difference in droplet volume, liquid-gas surface area and contact angle, near-identical peak and average heat fluxes are noted for both cases, due to the similar CLD. This shows that, for a given heated substrate-liquid combination, the conjugate heat transfer strongly depends on the droplet base radius and not on the geometric, thermal and wetting condition of the droplet. This being the case, it seems plausible to characterize the order of magnitude of conjugate heat transfer for a given droplet-substrate combination in terms of a lateral conduction Biot number, $B i=h L^{2} / k t$, where $h$ and $L$ are the characteristic heat transfer coefficient and characteristic length of the contact line region respectively, and $k$ and $t$ are the thermal conductivity and the thickness of the substrate.

\section{Conclusion}

The geometric properties and heat flux distributions to a hydrophilic evaporating droplet were characterized using thermal imaging and droplet shape analysis. These results were compared and contrasted with that of a superhydrophobic evaporating droplet over their respective evaporation periods. The hydrophilic droplet evaporated initially in the $\mathrm{CCR}$ regime, before transitioning to the mixed-mode regime once the contact angle decreased to the receding contact angle. The peak heat flux was noted at the contact line region for the entire life of the droplets. The average heat flux was noted to increase only marginally in the CCR regime, and this was as a result of the small yet monotonic increase in the contact line heat flux. During the CCR mode of evaporation, the peak heat flux was notably lower than that associated with a superhydrophobic droplet of similar volume. Once the contact line unpinned, the evaporation proceeded in a similar manner to that of the superhydrophobic droplet. Both the peak and average heat flux increased with escalating CLD. The total thermal power dissipated to the hydrophilic droplet decreased as the mixed-mode evaporation continued. The average heat flux density into the droplet was shown to be proportionally dependent on the CLD.

Comparing the superhydrophobic and hydrophilic droplet directly for similar volumes showed that the hydrophilic droplet dissipated twice the total power due to its larger solid-liquid surface area and total contact line length. This 
is despite having a lower peak and average heat flux. Contrasting the superhydrophobic and hydrophilic droplets for different and similar CLD highlights the importance of CLD in droplet evaporation. In particular, for similar working fluids and diffusion-based evaporation, it is concluded that the CLD largely dictates the relative proportion of the total heat transferred to the ambient via the low thermal resistance contact line region, and that for a given substrate the conjugate heat transfer depends much more on the CLD compared to the droplet contact angle or liquid-gas surface area. Future work will explore the impact of electric fields and gravity on the heat transfer to evaporating hydrophobic and hydrophilic droplets.

\section{Acknowledgements}

The authors would like to acknowledge the financial support of the Irish Research Council under Grant No. $\mathrm{RS} / 2012 / 86$.

\section{References}

[1] P. A. Raghupathi, S. G. Kandlikar, Contact line region heat transfer mechanisms for an evaporating interface, International Journal of Heat and Mass Transfer 95 (2016) 296-306.

[2] S. A. Putnam, A. M. Briones, J. S. Ervin, M. S. Hanchak, L. W. Byrd, J. G. Jones, Interfacial heat transfer during microdroplet evaporation on a laser heated surface, International Journal of Heat and Mass Transfer 55 (23) (2012) 6307-6320.

[3] W. Deng, A. Gomez, Electrospray cooling for microelectronics, International Journal of Heat and Mass Transfer 54 (11) (2011) 2270-2275.

[4] T. J. Hartranft, G. S. Settles, Sheet atomization of nonnewtonian liquids, Atomization and Sprays 13 (2\&3).

[5] M. Schena, D. Shalon, R. W. Davis, P. O. Brown, Quantitative monitoring of gene expression patterns with a complementary DNA microarray, Science 270 (5235) (1995) 467.

[6] V. Dugas, J. Broutin, E. Souteyrand, Droplet evaporation study applied to DNA chip manufacturing, Langmuir 21 (20) (2005) 9130-9136.

[7] A. Boutier, Laser Metrology in Fluid Mechanics: Granulometry, Temperature and Concentration Measurements, John Wiley \& Sons, 2013.

[8] G. Li, S. M. Flores, C. Vavilala, M. Schmittel, K. Graf, Evaporation dynamics of microdroplets on self-assembled monolayers of dialkyl disulfides, Langmuir 25 (23) (2009) 13438-13447.

[9] J. Kim, Spray cooling heat transfer: the state of the art, International Journal of Heat and Fluid Flow 28 (4) (2007) 753-767.

[10] S. A. Putnam, A. M. Briones, L. W. Byrd, J. S. Ervin, M. S. Hanchak, A. White, J. G. Jones, Microdroplet evaporation on superheated surfaces, International Journal of Heat and Mass Transfer 55 (21) (2012) 5793-5807.

[11] R.-H. Chen, T. X. Phuoc, D. Martello, Effects of nanoparticles on nanofluid droplet evaporation, International Journal of Heat and Mass Transfer 53 (19) (2010) 3677-3682.

[12] J. Park, J. Moon, Control of colloidal particle deposit patterns within picoliter droplets ejected by ink-jet printing, Langmuir 22 (8) (2006) 3506-3513.

[13] D. Brutin, Droplet Wetting and Evaporation: From Pure to Complex Fluids, Academic Press, 2015.

[14] M. Gibbons, A. Robinson, Heat transfer characteristics of single cone-jet electrosprays, International Journal of Heat and Mass Transfer 113 (2017) 70-83.

[15] M. Gibbons, P. D. Marco, A. Robinson, Local heat transfer to an evaporating superhydrophobic droplet, International Journal of Heat and Mass Transfer 121 (2018) 641 - 652. doi:https: //doi.org/10.1016/j.ijheatmasstransfer.2018.01.007.

URL http://www.sciencedirect.com/science/article/pii/ S0017931017332866

[16] A. L. Karchevsky, I. V. Marchuk, O. A. Kabov, Calculation of the heat flux near the liquid-gas-solid contact line, Applied Mathematical Modelling 40 (2) (2016) 1029-1037.

[17] J. N. Israelachvili, Intermolecular and surface forces, Academic press, 1992.

[18] M. Gibbons, C. Howe, P. Di Marco, A. Robinson, Local heat transfer to an evaporating sessile droplet in an electric field, in: Journal of Physics: Conference Series, Vol. 745, IOP Publishing, 2016, p. 032066.

[19] O. Raghu, J. Philip, Thermal properties of paint coatings on different backings using a scanning photo acoustic technique, Measurement Science and Technology 17 (11) (2006) 2945.

[20] S. Siedel, S. Cioulachtjian, A. J. Robinson, J. Bonjour, Integral momentum balance on a growing bubble, Physics of Fluids 25 (12) (2013) 123301.

[21] L. Kirkup, R. B. Frenkel, An Introduction to Uncertainty in Measurement: Using the GUM (Guide to the Expression of Uncertainty in Measurement), Cambridge University Press, 2006.

[22] H. Hu, R. G. Larson, Marangoni effect reverses coffee-ring depositions, The Journal of Physical Chemistry B 110 (14) (2006) 7090-7094.

[23] R. G. Larson, Transport and deposition patterns in drying sessile droplets, AIChE Journal 60 (5) (2014) 1538-1571.

[24] S. Dash, S. V. Garimella, Droplet evaporation dynamics on a superhydrophobic surface with negligible hysteresis, Langmuir.

[25] E. F. Crafton, W. Z. Black, Heat transfer and evaporation rates of small liquid droplets on heated horizontal surfaces, International Journal of Heat and Mass Transfer 47 (6) (2004) 11871200.

[26] E. Y. Gatapova, A. A. Semenov, D. V. Zaitsev, O. A. Kabov, Evaporation of a sessile water drop on a heated surface with controlled wettability, Colloids and Surfaces A: Physicochemical and Engineering Aspects 441 (2014) 776-785.

[27] K. Gleason, S. A. Putnam, Microdroplet evaporation with a forced pinned contact line, Langmuir 30 (34) (2014) 1054810555.

[28] R. Mollaret, K. Sefiane, J. R. Christy, D. Veyret, Experimental and numerical investigation of the evaporation into air of a drop on a heated surface, Chemical Engineering Research and Design 82 (4) (2004) 471-480.

[29] W. Xu, R. Leeladhar, Y. T. Kang, C.-H. Choi, Evaporation kinetics of sessile water droplets on micropillared superhydrophobic surfaces, Langmuir 29 (20) (2013) 6032-6041. 